\title{
Jingling the bells of innovation since the 3rd of September 2013
}

\author{
João José Pinto Ferreira \\ jjpf@fe.up.pt | INESC Technology and Science, Faculty of Engineering, University of Porto, Portugal \\ Anne-Laure Mention \\ anne-laure.mention@rmit.edu.au |RMIT University, Australia \\ Marko Torkkeli \\ marko.torkkeli@lut.fi | Lappeenranta University of Technology, Finland
}

\section{End of Year Message}

J ingling the bells of innovation since the 3rd of September 2013, and

O n a purposeful move to further discussion,

$\mathbf{U}$ nder a new umbrella of change. JIM was born from a

$\mathbf{R}$ eal need to reward multidisciplinary research! Like

$\mathbf{N}$ ever before, 2020 was a year far from any other in our lifetime.

A 11 around the world, COVID-19 has set the tune for destroyed

L ivelihoods, bracing for surviving. . .

O ne more hard day,

F eeling many times powerless, against a pervasive and powerful virus! 
I nnovation, however,

$\mathbf{N}$ ot only did it not stop, but it also accelerated!

$\mathbf{N}$ umerous examples demonstrate

O ngoing social, organizational and technological innovations! As

V ials entered our lexicon through daily news, and

A s multiple fields of engineering, medical and social sciences

I ntegrated knowledge to produce

O ff-beat solutions to problems, on the way,

$\mathbf{N}$ o relevant idea was spared exploration!

$\mathbf{M}$ ore innovation is currently unfolding...

A s we go, we will welcome your contributions!

$\mathbf{N}$ ot only as research papers, but also

A s letters and book reviews!

G oing forward, as we

E xplore possibilities and

$\mathbf{M}$ aximise value creation,

E xpect outstanding solutions! Along the way, never stop looking for

$\mathbf{N}$ ovel social, organizational and

T echnological innovation addressing the UN Sustainable Development Goals!

Innovatively yours,

João José Pinto Ferreira, Anne-Laure Mention, Marko Torkkeli

Founding Editors 